\title{
Multilayer scaffolds in orthopaedic tissue engineering
}

\author{
Kivanc Atesok • M. Nedim Doral · Jon Karlsson • Kenneth A. Egol • Laith M. Jazrawi • \\ Paulo G. Coelho $\cdot$ Amaury Martinez $\cdot$ Tomoyuki Matsumoto $\cdot$ Brett D. Owens · \\ Mitsuo Ochi $\cdot$ Shepard R. Hurwitz $\cdot$ Anthony Atala $\cdot$ Freddie H. Fu $\cdot$ Helen H. Lu $\cdot$ Scott A. Rodeo
}

Received: 29 June 2014 / Accepted: 18 November 2014

(C) European Society of Sports Traumatology, Knee Surgery, Arthroscopy (ESSKA) 2014

\begin{abstract}
Purpose The purpose of this study was to summarize the recent developments in the field of tissue engineering as they relate to multilayer scaffold designs in musculoskeletal regeneration.

Methods Clinical and basic research studies that highlight the current knowledge and potential future applications of the multilayer scaffolds in orthopaedic tissue engineering were evaluated and the best evidence collected. Studies were divided into three main categories based on tissue
\end{abstract}

K. Atesok $\cdot$ S. A. Rodeo $(\bowtie)$

Sports Medicine and Shoulder Service, Hospital for Special Surgery, 525 East 71st Street, New York, NY 10021, USA e-mail: RodeoS@HSS.edu

\section{K. Atesok}

e-mail: kivanc.atesok@utoronto.ca

\section{N. Doral}

Department of Orthopaedics and Traumatology, Hacettepe University School of Medicine, 06100 Sihhiye, Ankara, Turkey

\section{J. Karlsson}

Department of Orthopaedics, Sahlgrenska University Hospital, Sahlgrenska Academy, Gothenburg University, 43180 Molndal, Sweden

\section{K. A. Egol}

Department of Orthopaedic Surgery, NYU Hospital for Joint Diseases, 303 2nd Avenue, New York, NY 10003, USA

\section{M. Jazrawi · A. Martinez}

Center for Musculoskeletal Care, NYU Hospital for Joint

Diseases, 333 east 38th street, New York, NY 10016, USA

\section{P. G. Coelho}

Department of Periodontology and Implant Dentistry, New York University College of Dentistry, 345 east 24th street Room 804s, New York, NY 10010, USA types and interfaces for which multilayer scaffolds were used to regenerate: bone, osteochondral junction and tendon-to-bone interfaces.

Results In vitro and in vivo studies indicate that the use of stratified scaffolds composed of multiple layers with distinct compositions for regeneration of distinct tissue types within the same scaffold and anatomic location is feasible. This emerging tissue engineering approach has potential applications in regeneration of bone defects, osteochondral

\section{T. Matsumoto}

Department of Orthopaedic Surgery, Kobe University Graduate School of Medicine, Chuo-ku, Kobe 650-0017, Japan

\section{B. D. Owens}

Orthopaedic Surgery Service, Keller Army Hospital, 900

Washington Rd, West Point, New York, NY 10996, USA

M. Ochi

Department of Orthopaedic Surgery, Graduate School of Biomedical Sciences, Hiroshima University, 1-2-3 Kasumi, Minamimi-ku, Hiroshima 734-8551, Japan

S. R. Hurwitz

Department of Orthopaedic Surgery, University of North Carolina, Chapel Hill, NC, USA

S. R. Hurwitz

American Board of Orthopaedic Surgery, 400 Silver Cedar Court, Chapel Hill, NC 27514, USA

A. Atala

Wake Forest Institute for Regenerative Medicine, Wake Forest University School of Medicine, Winston-Salem, NC 27157, USA

F. H. Fu

Department of Orthopaedic Surgery, University of Pittsburgh School of Medicine, 3471 Fifth Avenue, Suite 1011, Pittsburgh, PA 15213, USA 
lesions and tendon-to-bone interfaces with successful basic research findings that encourage clinical applications.

Conclusions Present data supporting the advantages of the use of multilayer scaffolds as an emerging strategy in musculoskeletal tissue engineering are promising, however, still limited. Positive impacts of the use of next generation scaffolds in orthopaedic tissue engineering can be expected in terms of decreasing the invasiveness of current grafting techniques used for reconstruction of bone and osteochondral defects, and tendon-to-bone interfaces in near future.

Keywords Orthopaedic tissue engineering - Interface tissue regeneration $\cdot$ Multilayer scaffolds $\cdot$ Multi-lineage cell differentiation

\section{Introduction}

Tissue engineering or regenerative medicine is an emerging multidisciplinary field involving biology, medicine and engineering which has started to revolutionize ways to improve health and quality of life for millions of people worldwide by restoring, maintaining or enhancing tissue healing and organ function [33]. In the context of tissue engineering, the term "scaffold" can be simply defined as an artificial structure used to support three-dimensional tissue formation. Cells and biological factors in regenerating tissues and/or organs are often implanted or "seeded" into this artificial structure that is of critical importance in recapitulating the in vivo milieu and allowing cells to influence the local microenvironment [24].

Living tissues are non-homogenous entities which are composed of biologically and functionally different layers that coexist in hierarchy and harmony. Hence, using homogenous scaffolds to recapitulate heterogeneous living tissues will likely lead to suboptimal outcomes [14]. Over the last decade, interface tissue engineering has become an emerging branch of regenerative medicine, where the goal is to translate the non-homogenous multilayered structure of native tissues into scaffold formulations by producing new generation, stratified or gradient designs. In stratified designs, the scaffolds are segregated into two or more layers with abrupt changes in physical and chemical properties. In gradient designs, however, these changes are more gradual with no discrete segregation between layers.

Recognition of the necessity to integrate different tissue types, such as bone, cartilage and tendon, in order to successfully regenerate complex orthopaedic interfaces has been the driving force for the development of a new

H. H. Lu

Department of Biomedical Engineering, Columbia University, New York, NY 10016, USA generation of multilayer scaffolds in orthopaedic tissue engineering. Substantial research has been conducted and is underway to produce novel multilayer scaffold designs to mimic cartilage-to-bone or as well as ligament- and tendon-to-bone interfaces $[4,7,11,19,21,27,36]$. Current applications of next generation multiphase scaffold designs in orthopaedic tissue engineering can be summarized under three headings based on the anatomic site where they are used: bone, cartilage and tendon/ligament regeneration.

\section{Multilayer scaffolds in bone tissue engineering}

Bone is a dense connective tissue that presents mechanical, synthetic and metabolic functions [8]. Thus, its loss due to pathologic conditions or trauma has significant effects on patients' quality of life and requires regenerative techniques that can adequately result in full restoration of the affected bone form and function [13]. Regeneration of critically sized bone defects (defects that will not naturally heal to appropriately restore bone form and function) presents a significant challenge $[8,13]$. Currently, one of the most frequently utilized approaches to regenerate large bone defects relies on the placement of scaffolds under the premise that space maintenance will occur in tandem with new bone formation within the scaffold-filled defect $[4,10,22]$. Structure of bone tissue varies considerably throughout the skeleton based on anatomic location, both macroscopically and microscopically. Furthermore, the ultra-structure of bone tissue at a particular anatomic location is also organized non-homogenously and composed of different organic elements at different layers such as periosteum, cortex and medulla. Native bone tissue structure contains high levels of collagen as well as several non-collagenous materials, such as hydroxyapatite (HA). Although collagen-based scaffolds have been shown to have good biological effects based on their high porosity and permeability [35], they commonly suffer from poor mechanical properties and rapid enzymatic degradation, thereby limiting their use when high mechanical strength is required [30,32]. On the other hand, using the primary inorganic component of human bone matrix, HA, alone may also produce outcomes with limited mechanical function [16, 25]. Thus, a scaffold to engineer bone should ideally contain these key components together in a non-homogenously arranged three-dimensional (3D) structure [1].

The basic idea of creating multiphase scaffolds that can mimic native anatomy has found applications in regeneration of the periodontium complex [23]. Lee et al. used a 3D-printed multiphase scaffold for integrated periodontium regeneration. Three phases that contained microchannels with different sizes were designed for achieving integration of the scaffold into the cementum/dentin interface, periodontal ligament and alveolar bone [23]. Their results 
indicated that a single stem/progenitor cell population may differentiate into putative dentin/cementum, periodontal ligament and alveolar bone by such a scaffold's biophysical properties and spatially released bioactive cues.

In a recent study, Ding et al. [11] reported on a biphasic scaffold which consisted of polylactic acid-coated polygly-

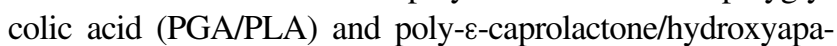
tite (PCL/HA) and was designed and used for regeneration of the goat femoral head. The content of PLA and HA was optimized to a proper ratio such that the scaffolds (PGA/PLA with $10 \%$ PLA and PCL/HA with $40 \mathrm{wt} \%$ HA, respectively) could achieve appropriate stiffness $(5.1 \pm 1.0 \mathrm{MPa}$ for PGA/PLA and $57.9 \pm 5.7 \mathrm{MPa}$ for PCL/HA) which was more conducive to articular cartilage and bone regeneration, respectively. The authors employed computer-aided design and manufacturing technology to fabricate the biphasic scaffolds into the desired shape and structure. Chondrocytes and bone marrow stromal cells (BMSCs) were seeded into the scaffolds for cartilage and bone regeneration, respectively. After 10 weeks of subcutaneous implantation in nude mice, the cell-scaffold constructs successfully regenerated goat femoral heads. The regenerated femoral heads presented a precise appearance in shape and size similar to that of native goat femoral heads with a smooth, continuous, avascular and homogeneous cartilage layer on the surface and stiff bone-like tissue in the microchannels of PCL/HA scaffold. Additionally, histological examination of the regenerated cartilage and bone showed typical histological structures and biophysical properties similar to those of native bone with specific matrix deposition and a well-integrated osteochondral interface [11].

While substantial basic and clinical research has been performed regarding the classic natural and synthetic scaffold materials presented thus far, it is clear that singlematerial scaffolds in large bone defects will not always result in full restoration of bone form and function. Designing scaffolds for bone regeneration that can combine desired mechanical strength and integrity with osteogenic/ conductive/inductive properties, while fitting well into the 3D anatomic location they are intended for, still requires extensive research. However, with the rapid evolution of portable 3D manufacturing technology which enables precise 3D bioprinting of scaffolds according to the particular patient anatomy [6], it is foreseen that defect-specific custom assembling of scaffolds made of multiple structural and biological materials will represent far less of a challenge than it currently represents.

\section{Multilayer scaffolds in cartilage tissue engineering}

Theoretically, the osteochondral junction can be divided into four layers depending on their different characteristics, including cell types, matrix composition, collagen fibre orientation and mechanical properties (Fig. 1): (1) superficial zone has fewer chondrocytes, higher water content and collagen fibres parallel to the joint surface [12, 28]. (2) Intermediate (middle) zone has larger collagen fibres interlacing with each other and cells with higher ECM production $[12,28]$. (3) Deep zone has tightly packed fibres perpendicular to the joint surface, active cells and lower water content [28]. (4) Calcified zone or "subchondral bone" is where the transition from soft to stiff subchondral bone occurs, responsible for firmly attaching the noncalcified cartilage to the underlying subchondral bone [18]. The first three layers consist of mostly collagen type II ( $90 \%$ ), glycosaminoglycans, cells and water; the last layer consists of collagen type I and type X, osteocalcin and hydroxyapatite. The cellular content of this junction has a transition from chondrocytes in the superficial layers to osteoblasts and osteoclasts in the subchondral bone, with some overlap in function and characteristics. For instance, hypertrophic chondrocyte-like cells are surrounded by a bone-like ECM in the calcified zone [18, 34].

The complexity of the osteochondral junction has led to the development of multilayer scaffolds with specific biological and mechanical properties to ideally promote individual growth of both cartilage and bone layers within a single integrated implant [17, 34] (Figs. 2, 3, 4).

To mimic the native osteochondral junction, Chen et al. [7] prepared a biphasic scaffold. The upper layer was a collagen sponge with relatively low mechanical strength that was designed to regenerate cartilage layer. The lower layer was a composite sponge of poly(lactic-co-glycolic acid) (PLGA) with higher mechanical strength that was designed to promote bone growth. The authors used the biphasic scaffold for culture of canine bone marrow-derived mesenchymal stem cells (MSCs). They implanted the cell/scaffold construct in an osteochondral defect in the knee of a 1 -year-old beagle. Osteochondral tissue was regenerated 4 months after implantation, and cartilage- and bone-like tissues were formed in the respective layers.

Kon et al. [19] developed an osteochondral scaffold with three layers; the superficial cartilaginous layer consisted of Type I collagen only, while the intermediate transition layer was composed of a combination of Type I collagen (60\% of weight) and magnesium-hydroxyapatite (Mg-HA) (40\% of weight), and the lower bone layer was composed of less Type I collagen (30\% of weight) and predominantly Mg-HA (70 \% of weight). The authors used these scaffolds with or without autologous chondrocytes in a sheep femoral condyle osteochondral defect model and compared their results with the control animals where no scaffold was applied to the defects. Significantly better integration of the chondral surface and bone regeneration was observed in the groups that received scaffolds compared with controls. There was no difference in cartilage 


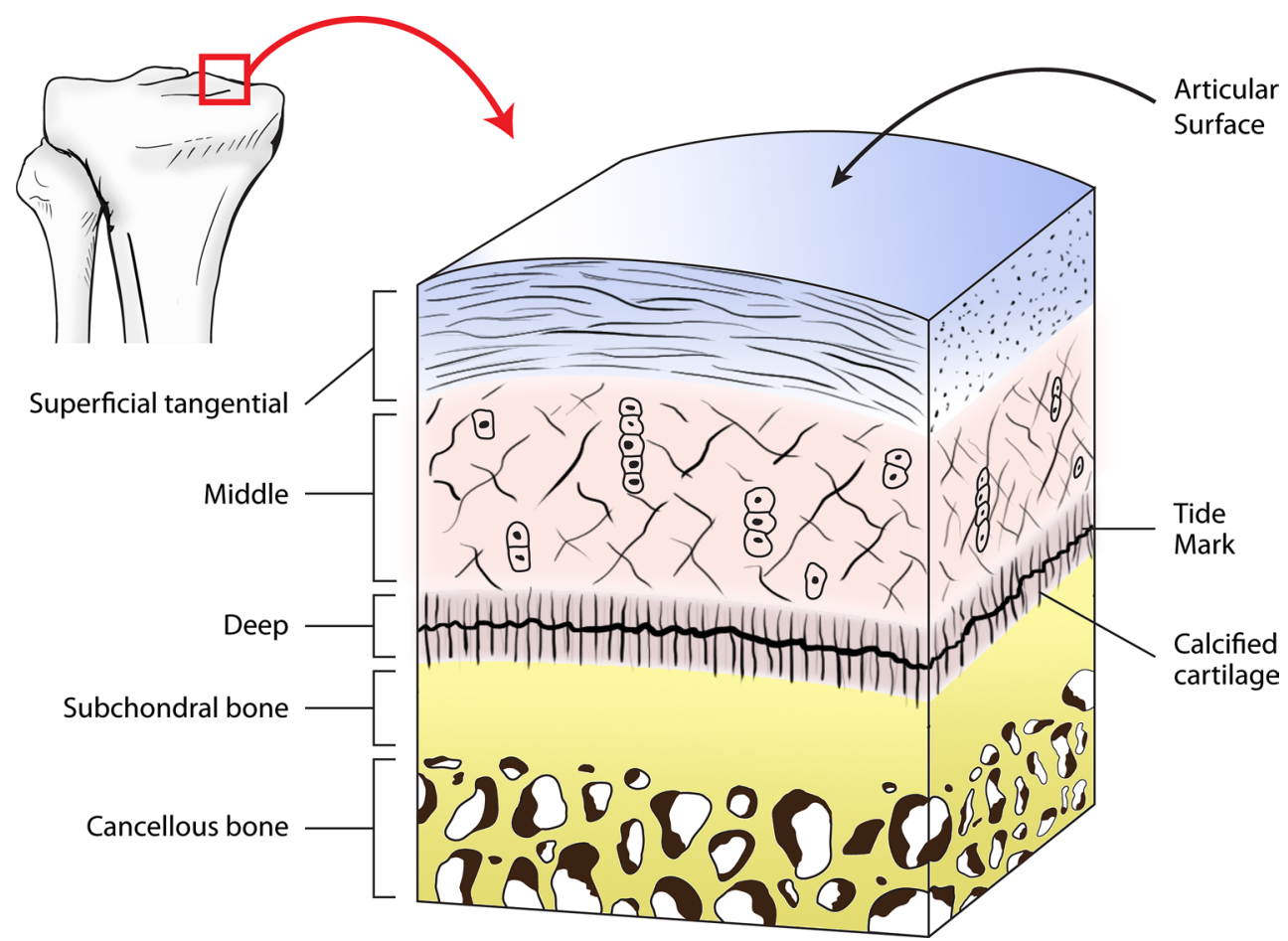

Fig. 1 The structure of osteochondral junction can be divided into four different zones based upon the cell morphology, matrix composition, collagen fibril orientation and mechanical properties. (1) Superficial zone has fewer chondrocytes and collagen fibres parallel to the joint surface. (2) Intermediate (middle) zone has larger collagen fibres interlacing with each other. (3) Deep zone has tightly packed fibres perpendicular to the joint surface, active cells and lower water content. Deep zone also includes tidemark (basophilic line which straddles the boundary between uncalcified and calcified cartilage). (4) Calcified zone or "subchondral bone" is where the transition from soft to stiff subchondral bone occurs, responsible for firmly attaching the noncalcified cartilage to the underlying subchondral bone
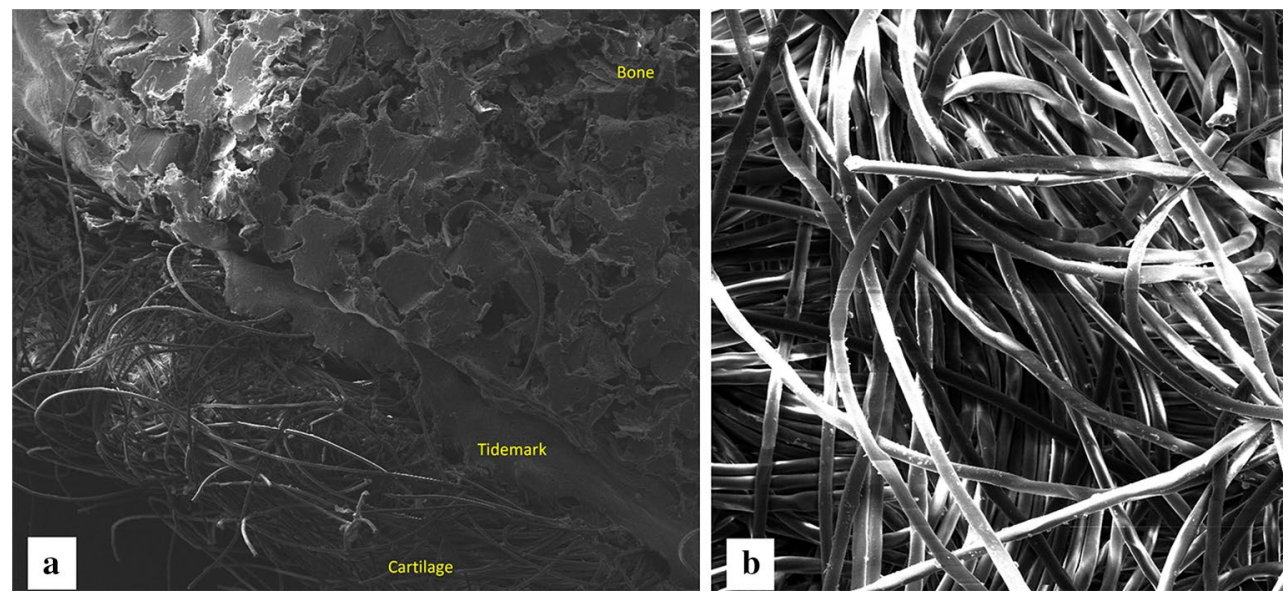

Fig. 2 Scanning electron micrographs of the composite osteochondral plug. Bone, tidemark and cartilage layers (a) and poly (glycolic acid) fibrils of cartilage zone (b). (Source: Aydin [3])

surface regeneration and defect filling between cell-seeded and cell-free scaffold groups. In this study, the use of triphasic scaffolds led to the regeneration of articular tissue with an ordered histoarchitecture. The same research group used multiphasic scaffolds for osteochondral defect regeneration in horses and obtained similar promising results with orderly regeneration of bone and hyaline-like cartilage tissue [20].

The safety and performance of new generation multilayered osteochondral scaffolds were tested in a clinical study including 30 patients with grade III-IV chondral and osteochondral lesions of the knee joint [21]. The 
Fig. 3 Micro-computerized tomography images of the osteochondral scaffold plugs. Polymeric layers (a) and calcium phosphate particles (b). (Source: Aydin [3])
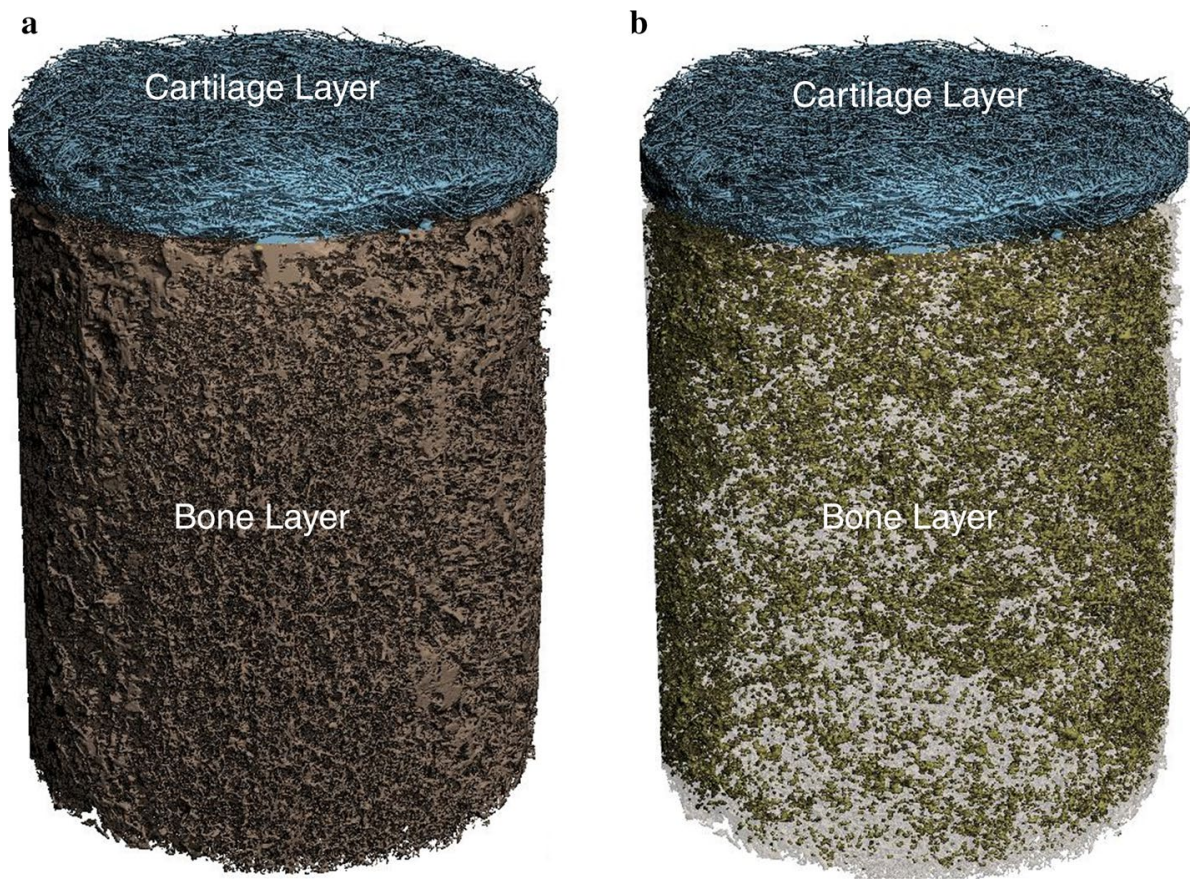

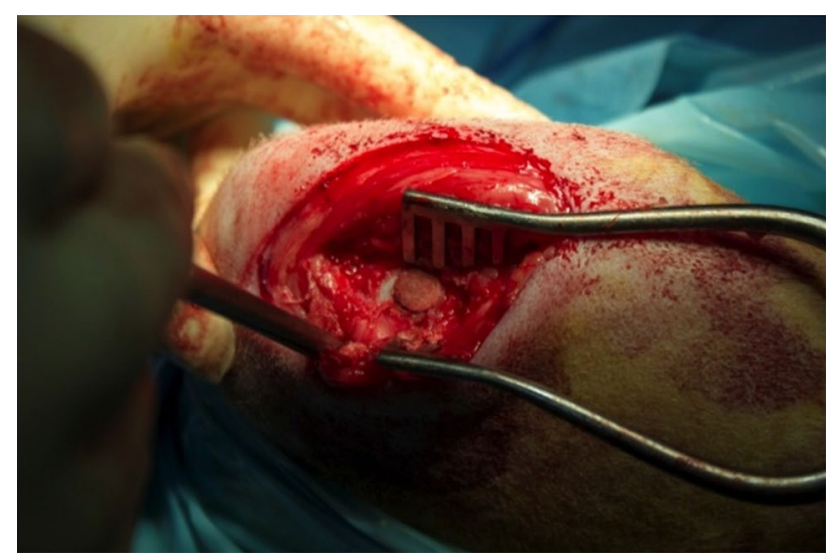

Fig. 4 Multilayered matrix after implantation into an osteochondral defect in bovine lateral femoral condyle. (Courtesy of Dr. H. M. Aydin, Institute of Science, Bioengineering Division, Hacettepe University, Beytepe 06800, Ankara, Turkey)

scaffold was trilayered with the same structural ingredients as described previously by Kon et al. [19]. The sites of the defects included medial and lateral femoral condyles, patellas, trochleas and tibial plateaus with an average defect size of $2.9 \mathrm{~cm}^{2}$. Twenty-eight patients were followed for 2 years and were clinically evaluated using the International Knee Documentation Committee (IKDC) and Tegner activity scale scores. At the final follow-up, magnetic resonance imaging evaluation was performed in 24 patients using magnetic resonance observation of cartilage repair tissue (MOCART) scoring system. IKDC and Tegner activity scores showed a statistically significant improvement
$(P<0.0005)$ from the preoperative level to the 1 - and 2-year follow-ups. However, level of sports activity was significantly lower with respect to preinjury level at 1and 2-year follow-up $(P=0.003$ and 0.007 , respectively). MOCART evaluation showed complete filling of the defect with integration of the graft in $70 \%$ of the patients. However, the subchondral lamina and bone were considered intact in a minority of cases (7 and $47 \%$, respectively). This study did not include a control group, and the followup was relatively short. Additionally, osteochondral defects were not standard in every patient in terms of location, size and depth. Furthermore, limited number of patients, differences between age and activity level, and associated injuries with additional surgeries might have confounded the results [21].

Based on animal models and limited clinical reports, osteochondral tissue engineering using multilayer scaffolds appears as a feasible and safe method with promising shortterm outcomes. It should be noted that no animal models or clinical studies compared the use of multilayer scaffolds with monolayer homogenous scaffolds in osteochondral defect regeneration, and data from randomized clinical trials are lacking.

\section{Multilayer scaffolds in tendon/ligament tissue engineering}

Tendon injuries are frequently encountered in orthopaedic clinical practice and cause substantial morbidity in sports and in routine daily activities. The anterior 
$\mathbf{a}$

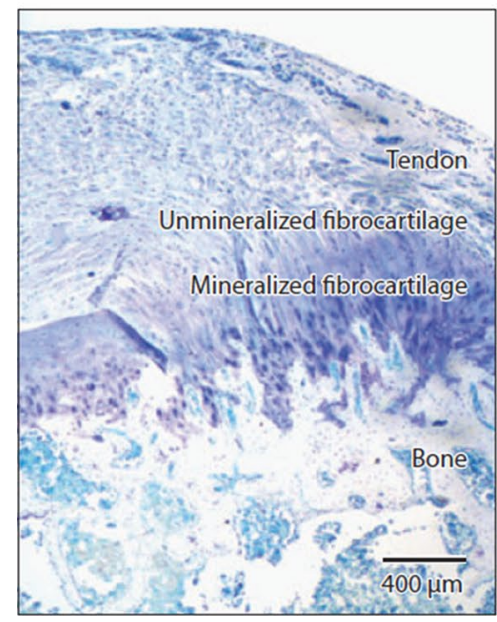

Fig. 5 a Tendon and $\mathbf{b}$ ligament attach to bone across a functionally graded fibrocartilaginous transition site (a toluidine blue-stained section from an adult rat supraspinatus tendon-to-bone insertion is b

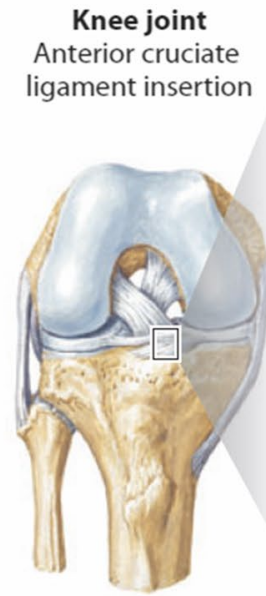

shown in panel a, and fast blue-stained section from a mature bovine anterior cruciate ligament insertion is shown in b). (Source: Lu and Thomopoulos [26])
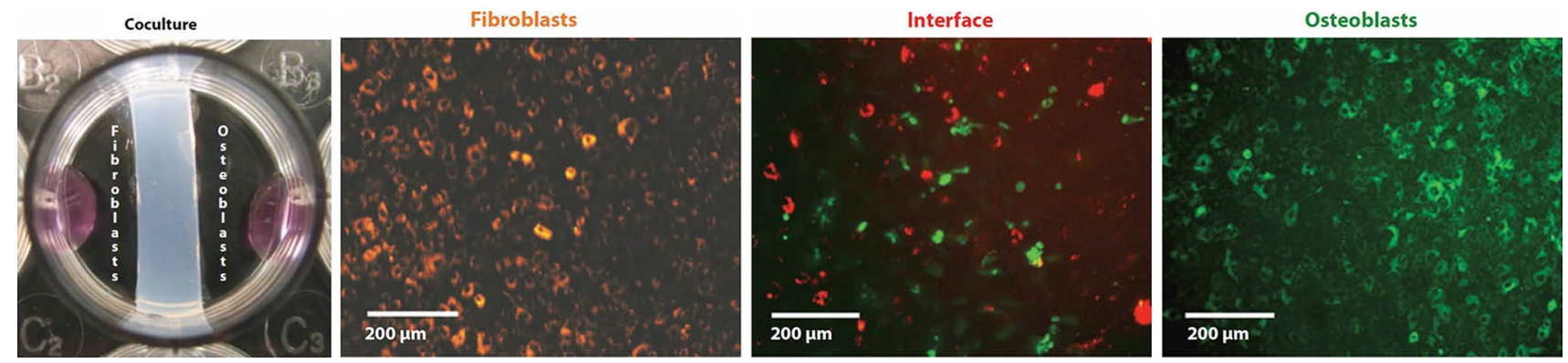

Fig. 6 Co-culture of fibroblasts and osteoblasts (left picture) exerts spatial control of cell distribution, resulting in an interface region that contains interacting osteoblasts and fibroblasts (right picture). (Source: Lu and Thomopoulos [26])

cruciate ligament (ACL) and rotator cuff (RC) are among the most commonly injured soft tissue structures. In addition to the importance of anatomical relocation of a tendon, morphological replication of the unique tendonto-bone insertion site is essential to achieve the desired material properties of the tendon-to-bone attachment site (Fig. 5) [2, 31].

The direct tendon-to-bone insertions, such as the insertions of the ACL and RC, are characterized by the presence of fibrocartilage tissue connecting these soft tissue structures to deeper layers of the bone [37]. The direct insertion site includes a transition zone that consists of four distinct tissue types: tendon/ligament, unmineralized fibrocartilage, mineralized fibrocartilage and bone [5, 9]. This multi-tissue transition (tendon/ligament, fibrocartilage and bone) represents a significant challenge in orthopaedic interface tissue engineering as several distinct yet contiguous tissue regions constitute this complex insertion site. The use of multilayer (stratified) biomimetic scaffolds therefore may be highly beneficial for reformation of the complex ligament-to-bone interface [29] (Fig. 6).

In a rat model study, Spalazzi et al. [36] reported the design and in vivo evaluation of a triphasic scaffold for regeneration of the ACL-to-bone interface (Phase A for the ligament region, Phase $\mathrm{B}$ for the interface and Phase $\mathrm{C}$ for the bone region). The researchers found that the triphasic scaffold supported multi-lineage cellular interactions as well as tissue infiltration and abundant matrix production in vivo. The results of this study demonstrated the feasibility of multi-tissue regeneration on a single graft by the use of multilayered scaffolds.

An in vitro study by He et al. [15] supported the feasibility of multilayer scaffolds designs in generating multilineage cellular and tissue environments. The researchers co-cultured bone marrow MSCs on a hybrid silk scaffold which included fibroblasts for ligament tissue differentiation on one end, HA with osteoblasts for bone tissue differentiation on the other end and MSCs in between. They 


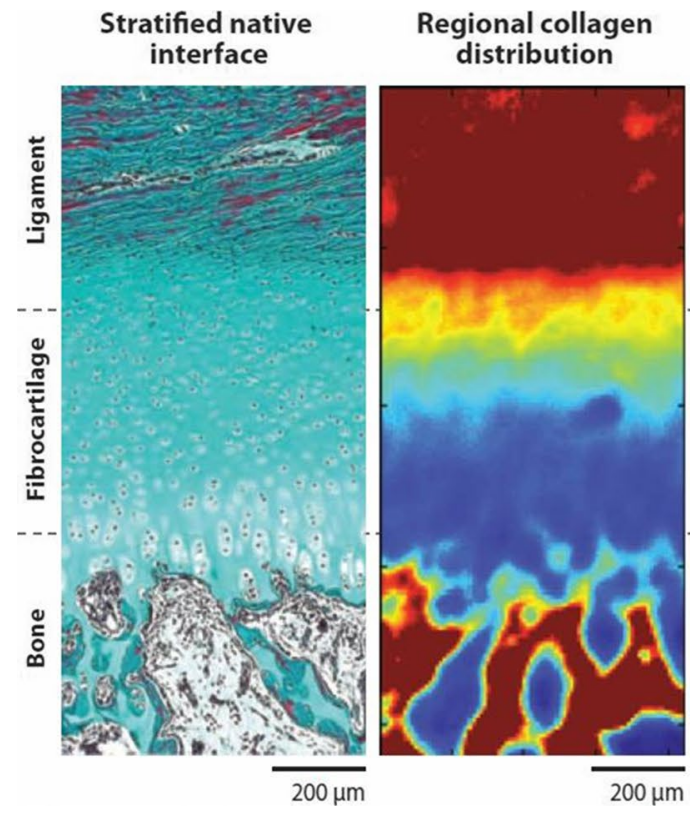

Fig. 7 Biomimetic strategy for engineering a ligament-to-bone interface. Inspired by the native enthesis, a stratified scaffold is designed to mimic the layered tissue regions progressing from ligament to fibrocartilage to bone. Spatial control over cell distribution
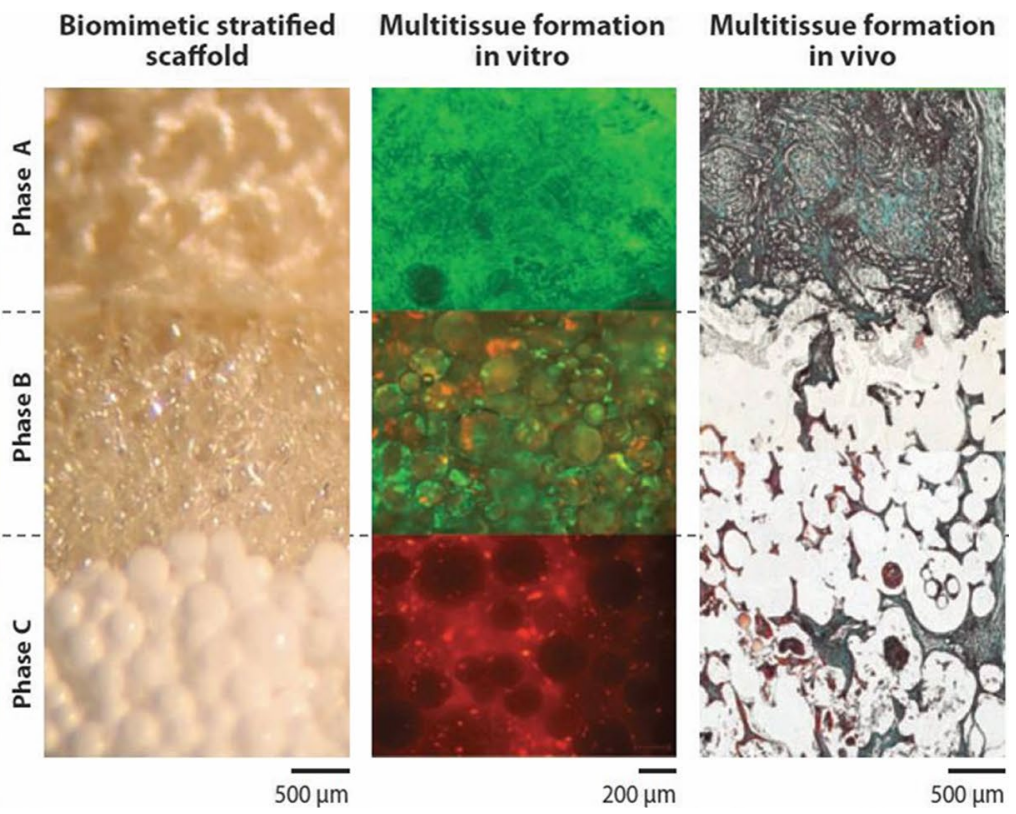

(fibroblasts, chondrocytes and osteoblasts) on the triphasic scaffold resulted in the formation of compositionally distinct yet structurally continuous tissue regions mimicking those found at the native ligament-to-bone insertion site. (Source: Lu and Thomopoulos [26])

Table 1 Summary of the type and evidence levels of the main studies from which data have been reported

\begin{tabular}{lllll}
\hline Tissue & References & Study type & Evidence level & Notes \\
\hline Bone & Ding et al. [11] & Animal model & N/A & Animals assigned to study or control group randomly \\
& Lee et al. [23] & Animal model & N/A & Study included in vitro and in vivo stages \\
Cartilage & Chen et al. [7] & Animal model & N/A & Assessment performed using SEM and histological staining \\
& Kon et al. [21] & Clinical study & Level 4 & Patients followed prospectively. No control group \\
& Kon et al. [19] & Animal model & N/A & Animals assigned to study or control group randomly \\
Tendon/ligament & He et al. [15] & In vitro study & N/A & $\begin{array}{l}\text { Proof-of-principle-type study } \\
\text { Concept review and expert opinion supported by published } \\
\end{array}$ \\
& Lu and Thomopoulos [26] & Review & Level 5 & evidence \\
& Spalazzi et al. [36] & Animal model & N/A & Assessment performed using histological staining, micro-CT and \\
& & &
\end{tabular}

N/A not applicable, SEM scanning electron microscopy

observed that bone marrow MSCs co-cultured between fibroblasts and osteoblasts had differentiated into a fibrocartilaginous lineage with a gradual transition from an uncalcified to a calcified region mimicking the native tendon-to-bone interface (Fig. 7). These results are consistent with those of Spalazzi et al. [36] and suggest that regeneration of tendon-to-bone healing can be achieved using a single scaffold with separate layers for multi-tissue transition from tendon/ligament to fibrocartilage and bone.

Regeneration of tissue-to-tissue interfaces through interface tissue engineering using multilayer scaffolds represents a promising strategy for achieving integrative soft tissue repair. It can be anticipated that these efforts will lead to the development of a new generation of functional fixation devices for orthopaedic repairs as well as augmenting the clinical translation potential of tissue engineered orthopaedic grafts [27]. However, substantial research with larger animal models and, eventually, randomized controlled human trials are required before these novel approaches can find clinical applications.

\section{Current limitations and future perspectives}

Through the aforementioned studies as well as other published work, it has been demonstrated that multilayered 
scaffolds are optimal for multi-tissue regeneration, and stem cell differentiation on these complex scaffold may be further harnessed in order to enable complex tissue formation and ease clinical translation of these grafts. Biomimetic gradient scaffolds are also promising approaches, especially if the gradient resolution is refined to physiologically relevant levels as fabrication techniques advance. Finally, scaling up of these complex scaffolds for multitissue regeneration represents another essential step towards clinical utilization which may offer the potential to decrease invasiveness associated with surgical procedures such as autologous grafting for tendon-to-bone interface or osteochondral defect reconstruction.

\section{Conclusions}

Multilayer scaffolds offer a promising strategy in musculoskeletal tissue engineering by allowing for the possibility to regenerate distinct tissue types such as bone, cartilage and tendon/ligament, simultaneously within the same construct and anatomic location. These novel scaffold designs which bridge distinct types of tissues through interface tissue engineering are instrumental for the development of integrated musculoskeletal tissue systems with biomimetic complexity and functionality [26, 27]. Current evidence does not include results from studies comparing multilayer scaffolds with single layer homogenous designs, and randomized clinical trials are lacking (Table 1).

\section{References}

1. Akkouch A, Zhang Z, Rouabhia M (2011) A novel collagen/ hydroxyapatite/poly(lactide-co- $\varepsilon$-caprolactone) biodegradable and bioactive $3 \mathrm{D}$ porous scaffold for bone regeneration. J Biomed Mater Res A 96(4):693-704

2. Atesok K, Fu FH, Wolf MR, Ochi M, Jazrawi LM, Doral MN, Lubovitz J, Rodeo S (2014) Augmentation of tendon-to-bone healing. J Bone Joint Surg Am 96(6):513-521

3. Aydin HM (2011) A three-layered osteochondral plug: structural, mechanical, and in vitro biocompatibility analysis. Adv Eng Mater. 13(12):B511-B517

4. Billstrom GH, Blom AW, Larsson S, Beswick AD (2013) Application of scaffolds for bone regeneration strategies: current trends and future directions. Injury 44(Suppl 1):S28-S33

5. Brophy RH, Kovacevic D, Imhauser CW, Stasiak M, Bedi A, Fox AJ, Deng XH, Rodeo SA (2011) Effect of short-duration lowmagnitude cyclic loading versus immobilization on tendon-bone healing after ACL reconstruction in a rat model. J Bone Joint Surg Am 93(4):381-393

6. Butscher A, Bohner M, Hofmann S, Gauckler L, Muller R (2011) Structural and material approaches to bone tissue engineering in powder-based three-dimensional printing. Acta Biomater 7(3):907-920

7. Chen G, Sato T, Tanaka J, Tateishi T (2006) Preparation of a biphasic scaffold for osteochondral tissue engineering. Mater Sci Eng C 26:118-123
8. Clarke B (2008) Normal bone anatomy and physiology. Clin J Am Soc Nephrol 3(Suppl 3):S131-S139

9. Cooper RR, Misol S (1970) Tendon and ligament insertion. A light and electron microscopic study. J Bone Joint Surg Am 52(1): $1-20$

10. Crowley C, Wong JM, Fisher DM, Khan WS (2013) A systematic review on preclinical and clinical studies on the use of scaffolds for bone repair in skeletal defects. Curr Stem Cell Res Ther 8(3):243-252

11. Ding C, Qiao Z, Jiang W, Li H, Wei J, Zhou G, Dai K (2013) Regeneration of a goat femoral head using a tissue-specific, biphasic scaffold fabricated with CAD/CAM technology. Biomaterials 34(28):6706-6716

12. Franzen A, Inerot S, Hejderup SO, Heinegard D (1981) Variations in the composition of bovine hip articular cartilage with distance from the articular surface. Biochem J 195:535

13. Giannotti S, Bottai V, Dell'osso G, Pini E, De Paola G, Bugelli G et al (2013) Current medical treatment strategies concerning fracture healing. Clin Cases Miner Bone Metab 10(2):116-120

14. Han LH, Suri S, Schmidt CE, Chen S (2010) Fabrication of three-dimensional scaffolds for heterogeneous tissue engineering. Biomed Microdevices 12(4):721-725

15. He P, Ng KS, Toh SL, Goh JC (2012) In vitro ligament-bone interface regeneration using a trilineage coculture system on a hybrid silk scaffold. Biomacromolecules 13(9):2692-2703

16. Ishihara K, Arai H, Nakabayashi N, Morita S, Furuya K (1992) Adhesive bone cement containing hydroxyapatite particle as bone compatible filler. J Biomed Mater Res 26:937-945

17. Jiang J, Tang A, Ateshian GA, Guo XE, Hung CT, Lu HH (2010) Bioactive stratified polymer ceramic-hydrogel scaffold for integrative osteochondral repair. Ann Biomed Eng 38(6):2183-2196

18. Keeney M, Pandit A (2009) The osteochondral junction and its repair via bi-phasic tissue engineering scaffolds. Tissue Eng Part B Rev 15(1):55-73

19. Kon E, Delcogliano M, Filardo G, Fini M, Giavaresi G, Francioli S, Martin I, Pressato D, Arcangeli E, Quarto R, Sandri M, Marcacci M (2010) Orderly osteochondral regeneration in a sheep model using a novel nano-composite multilayered biomaterial. J Orthop Res 28(1):116-124

20. Kon E, Mutini A, Arcangeli E, Delcogliano M, Filardo G, Nicoli Aldini N, Pressato D, Quarto R, Zaffagnini S, Marcacci M (2010) Novel nanostructured scaffold for osteochondral regeneration: pilot study in horses. J Tissue Eng Regen Med 4(4):300-308

21. Kon E, Delcogliano M, Filardo G, Busacca M, Di Martino A, Marcacci M (2011) Novel nano-composite multilayered biomaterial for osteochondral regeneration: a pilot clinical trial. Am J Sports Med 39(6):1180-1190

22. Kurien T, Pearson RG, Scammell BE (2013) Bone graft substitutes currently available in orthopaedic practice: the evidence for their use. Bone Joint J 95-B(5):583-597

23. Lee CH, Hajibandeh J, Suzuki T, Fan A, Shang P, Mao JJ (2014) Three-dimensional printed multiphase scaffolds for regeneration of periodontium complex. Tissue Eng Part A 20(7-8):1342-1351

24. Liu WF, Hui EE, Bhatia SN, Chen CS (2010) Engineering cellular microenvironments. In: Atala A, Lanza R, Thomson JA, Nerem RM (eds) Foundations of regenerative medicine: clinical and therapeutic applications. Academic Print by Elsevier, San Diego, Burlington, London, pp 284-302

25. Lopes MA, Monteiro FJ, Santos JD (1999) Glass-reinforced hydroxyapatite composites: fracture toughness and hardness dependence on microstructural characteristics. Biomaterials 20:2085-2090

26. Lu HH, Thomopoulos S (2013) Functional attachment of soft tissues to bone: development, healing, and tissue engineering. Annu Rev Biomed Eng 15:201-226 
27. Lu HH, Subramony SD, Boushell MK, Zhang X (2010) Tissue engineering strategies for the regeneration of orthopedic interfaces. Ann Biomed Eng 38(6):2142-2154

28. Madry H, van Dijk CN, Mueller-Gerbl M (2010) The basic science of the subchondral bone. Knee Surg Sports Traumatol Arthrosc 18(4):419-433

29. Moffat KL, Wang IN, Rodeo SA, Lu HH (2009) Orthopedic interface tissue engineering for the biological fixation of soft tissue grafts. Clin Sports Med 28(1):157-176

30. Moreau JL, Weir MD, Xu HH (2009) Self-setting collagencalcium phosphate bone cement: mechanical and cellular properties. J Biomed Mater Res A 91:605-613

31. Newsham-West R, Nicholson H, Walton M, Milburn P (2007) Long-term morphology of a healing bone-tendon interface: a histological observation in the sheep model. J Anat 210(3):318-327

32. Ng KW, Khor HL, Hutmacher DW (2004) In vitro characterization of natural and synthetic dermal matrices cultured with human dermal fibroblasts. Biomaterials 25:2807-2818
33. NIH Definition of tissue engineering/regenerative medicine. http://www.tissue-engineering.net/

34. Nooeaid P, Salih V, Beier JP, Boccaccini AR (2012) Osteochondral tissue engineering: scaffolds, stem cells and applications. J Cell Mol Med 16(10):2247-2270

35. O'Brien FJ, Harley BA, Yannas IV, Gibson L (2004) Influence of freezing rate on pore structure in freeze-dried collagen-GAG scaffolds. Biomaterials 25:1077-1086

36. Spalazzi JP, Dagher E, Doty SB, Guo XE, Rodeo SA, Lu HH (2008) In vivo evaluation of a multiphased scaffold designed for orthopaedic interface tissue engineering and soft tissue-to-bone integration. J Biomed Mater Res A 86(1):1-12

37. Woo S, Maynard J, Butler D, Lyon R, Torzilli P, Akeson W (1988) Ligament, tendon, and joint capsule insertions to bone. In: Woo SL-Y, Buckwalter JA (eds) Injury and repair of the musculoskeletal soft tissues. American Academy of Orthopaedic Surgery, Park Ridge, pp 133-166 